and evaluate the change of coronary and renal haemodynamic parameters on York pig model on AMI-ADHF.

Methods 10 York pigs were selected in this trial. LAD was occluded by balloon which was placed on mid-distal for 30-45 min until ST segment elevation for $15 \mathrm{~min}$. After deflating the balloon, the sterile microembolus were injected into LAD intermittently and injecting stopped until LVEDP $>18 \mathrm{~mm} \mathrm{Hg}$. The coronary pressure and flow velocity were recorded simultaneously at baseline, instant, $30 \mathrm{~min}$ and 60 min after AMI-ADHF model success. Average peak velocity (APV), coronary resistance and coronary flow reserve were obtained at basic and maximal hyperaemia. Renal artery angiography was performed and renal artery diameter and pressure was measured. Doppler flowire was placed in renal artery for measure of APV of renal artery $\left(\mathrm{APV}_{\mathrm{ra}}\right)$, renal artery vascular resistance and renal blood flow. At the same time ECG were recorded per $15 \mathrm{~min}$. CK-MB and TnI were measured in all York pigs at baseline, $2 \mathrm{~h}, 4 \mathrm{~h}, 8 \mathrm{~h}, 12 \mathrm{~h}$ and $24 \mathrm{~h}$ after reperfusion. Cardiac ultrasonograph was performed to evaluate the heart function.

Results (1) According to the standards of AMI-ADHF, eight models were successfully established. (2) Basic and hyperaemia APV were all decreased significantly, they were lower than that of before AMIADHF. coronary resistance was increased after AMI-ADHF model success. coronary flow reserve was lower than that of baseline. (3) Renal artery pressure has increased trend than baseline. renal artery vascular resistance was increased, APVr and renal blood flow was decreased than baseline.

Conclusion A stable experimental method of York pig model of AMIADHF was established successfully by coronary occlusion with balloon and injecting of microembolus in LAD. The method had advantages of closed chest, higher succeed rate and stablility to those of drug induced, tachycardia-pacing induced, coronary artery ligation induced or microsphere injection alone.

\section{e0659 THE EFFECT AND SAFETY OF TIROFIBAN COMBINED CLOPIDOGREL IN AMI PATIENTS WITH PCI VIA TRANSRADIAL APPROACH}

doi:10.1136/hrt.2010.208967.659

Fu Xianghua, Hao Oingqing, Fan Weize, Zhang Jing, Gu Xinshun, Jiang Yunfa, Hao Guozhen. The 2nd Hospital of Hebei Medical University, Shijiazhuang, Hebei, China

Objective To evaluate the effect and safety of clopidogrel plus tirofiban in percutaneous coronary intervention (PCI) of acute myocardial infarction via transradial approach.

Methods A total of 104 patients with ST segment elevation myocardial infarction who were underwent emergency PCI were randomised into two groups: clopidogrel combined tirofiban group $(n=48)$ and clopidogrel group $(n=56)$. Bolus tirofiban $(10 \mu \mathrm{g} / \mathrm{kg})$ was infused in $3 \mathrm{~min}$ before PCI and then $0.15 \mu \mathrm{g} / \mathrm{kg} / \mathrm{min}$ continued pumping for $24 \mathrm{~h}$ in the clopidogrel combined tirofiban group, while saline was used in the same way in the clopidogrel group. The TIMI flow grade in IRA before PCI, after the first guide wire getting through and instant post-PCI were recorded and compared between the two groups. The myocardial blush grade post-PCI and heart function were analysed and compared between the two groups. Hemorrhagic complications and MACE in-hospital were monitored and followed up.

Results The percentage of TIMI flow grade 1 of IRA before PCI was higher in the clopidogrel combined tirofiban group than that in the clopidogrel group $(p<0.05)$. The percentage of TIMI flow grade 3 of IRA after the guide wire first crossing through the occlusion segment was higher in clopidogrel combined tirofiban group than that in the clopidogrel group $(\mathrm{p}<0.05)$. The percentage of TIMI flow grade 3 of IRA after PCI was also higher in clopidogrel combined tirofiban group than that in the clopidogrel group $(p<0.05)$. The corrected TIMI flow count (cTFC) in the clopidogrel combined tirofiban group was smaller to the clopidogrel group $(p<0.05)$. LVEF at 1 week after PCI in the tirofiban group was higher than that in the clopidogrel group $(p<0.01)$. There was no significant difference in hemorrhagic complications between the two groups. The incidence rate of MACE during in-hospital in the clopidogrel combined tirofiban group was lower compared to that in the clopidogrel group $(\mathrm{p}<0.05)$.

Conclusions Clopidogrel combined tirofiban used during the emergency PCI for AMI via transradial approach can quickly inhibit the platelet aggregation, decrease thrombosis event, improve blood flow of IRA and increase the perfusion of myocardial microcirculation as well preserve the function of left ventricle and reduce the incidence rate of MACE during in-hospital, while not associate with increasing the incidence rate of serious haemorrhage complications.

\section{e0660 OUALITY OF LIFE AFTER FOUR TIMES INTRAVENOUS INFUSION OF BONE MARROW MSCS FOR HEART FAILURE PATIENTS}

doi:10.1136/hrt.2010.208967.660

${ }^{1}$ Oi Chunmei, ${ }^{2} \mathrm{Hao} \mathrm{Ji},{ }^{2}$ Zhang Xueshan, ${ }^{2}$ Feng Jianqi, ${ }^{2}$ Diao Jun, ${ }^{2}$ Wu Weiheng. ${ }^{1}$ Department of Cardiology, The Second Hospital Affiliated to Xuzhou Medical College; ${ }^{2}$ Department of Cardiology, The Second Hospital Affiliated to Xuzhou Medical College

Objective Heart failure is a complex clinical syndrome that can result from any structural or functional heart disorder, so the patients have marked limitation of physical activity and poor life quality. Some experiments have tested the safety and efficacy of intravenously injection of MSCs to restore the heart function, the goal of this study is to verify patients will have a better quality of life after stem cell transplantation.

Methods The study involved 85 acute myocardial infarction patients with heart failure who had ejection fractions less than $45 \%$. Between May 2008 and January 2010, 43 consecutive heart failure patients underwent MSCs injcetion through vein for four times in the experimental group. The number of cells prior to injection was 41.6 \pm 34.7 million cells, another 42 heart failure patients were as control group. NYHA class, 6 min walk test, Brain natridiureticpeptide level, transthoracic echocardiography, single-photon emission CT are obtained prior to transplatation, the patients who did not received PCI examed by MRI. Quality of life evaluated by SF36. These tests are repeated at 6 months.

Results At six-month follow-up, transplantation of MSCs enhanced left-ventricular systolic function $(39.12 \pm 4.54) \%$ versus $(52.08 \pm 1.02) \%$, emission CT $(40.19 \pm 3.18) \%$ versus $(53.18 \pm 2.15) \%$, BNP declined $(429 \pm 39) \mathrm{pg} \cdot \mathrm{ml}^{-1}$ versus $(152 \pm 19) \mathrm{pg} \cdot \mathrm{ml}^{-1}$, quality of life of the stem cell transpaltation patients were significantly improved according to the assessment of physical functioning, role limitations due to physical health, bodily pain, general health perceptions and vitality, but the social functioning, role limitations due to emotional problems and mental health were not improved. NYHA class decreased $(2.08 \pm 0.25)$ versus $(1.85 \pm 0.38), 6 \mathrm{~min}$ walk test increased $(328.3 \pm 45.3)$ versus $(356.8 \pm 43.2)$. MRI examinantion also observed the decreased infarct size in the experimental group.

Conclusion Quality of life and ejection fraction were significantly improved after Bone marrow MSCs injected through four times intravenous infusion. 